\title{
Algèbres topologiques à idéaux à gauche fermés
}

\author{
par \\ R. Choukri, A. El Kinani et M. Oudadess (Rabat)
}

\begin{abstract}
We consider topological algebras in which all left ideals are closed. We show, in particular, that an $F$-algebra has this property if, and only if, it is a noetherian $Q$-algebra.
\end{abstract}

1. Introduction. Soit $A$ une algèbre topologique. Considérons la propriété suivante :

$\left(\mathrm{F}_{\mathrm{g}}\right) \quad$ Tout idéal à gauche de $A$ est fermé.

Il est bien connu, d'après un résultat de Sidney, qu'une algèbre de Banach vérifiant $\left(\mathrm{F}_{\mathrm{g}}\right)$ est de dimension finie. Ce résultat n'est pas valide dans le cas $m$-convexe. En effet, l'algèbre des séries formelles est un contreexemple. Un autre contre-exemple, qui est de plus semi-simple, est donné dans [3]. D'autre part, bien que la propriété $\left(\mathrm{F}_{\mathrm{g}}\right)$ soit de nature topologique, elle est caractérisée algébriquement pour une classe importante d'algèbres topologiques. En effet, nous montrons qu'une $F$-algèbre vérifie $\left(\mathrm{F}_{\mathrm{g}}\right)$ si, et seulement si, elle est une $Q$-algèbre noethérienne (théorème 2). Dans le cas Fréchet, moyennant un résultat d'Arens, une telle algèbre vérifie $\left(\mathrm{F}_{\mathrm{g}}\right)$ si, et seulement si, elle est noethérienne (théorème 3). Par ailleurs, nous montrons (théorème 4) qu'une algèbre commutative artinienne vérifie $\left(\mathrm{F}_{\mathrm{g}}\right)$ pour toute topologie d'algèbre. Ce "caractère topologique universel" caractérise l'artinianité dans la classe des algèbres commutatives noethériennes semisimples (théorème 5).

2. Préliminaires. Toutes les algèbres considérées sont supposées complexes et unitaires. Une algèbre $A$ est dite noethérienne à gauche si elle vérifie l'une des conditions équivalentes suivantes :

(i) toute suite croissante d'idéaux à gauche de $A$ est stationnaire;

(ii) toute famille non vide d'idéaux à gauche de $A$ admet un élément maximal ; 
(iii) tout idéal à gauche de $A$ est de type fini (i.e., il est engendré par un nombre fini de ses éléments).

L'algèbre $A$ est dite artinienne à gauche si la famille de ses idéaux à gauche satisfait la condition de la chaine descendante.

Une algèbre est dite topologique si elle est munie d'une topologie d'espace vectoriel pour laquelle le produit est séparément continu. Une telle algèbre est dite:

(i) une $Q$-algèbre si le groupe de ses éléments inversibles est ouvert;

(ii) une F-algèbre si elle est métrisable et complète;

(iii) une algèbre localement multiplicativement convexe (a.l.m.c.) si sa topologie peut être définie par une famille de semi-normes sousmultiplicatives.

Une a.l.m.c. qui est une $F$-algèbre est dite de Fréchet. Sa topologie peut être alors définie par une famille dénombrable de semi-normes sous-multiplicatives.

Dans la suite, $S_{0}^{1}$ (resp. $\left.S_{0}\right)$ désigne l'algèbre des suites complexes stationnaires (resp. nulles à partir d'un certain rang).

3. Condition $\left(\mathrm{F}_{\mathrm{g}}\right)$, noethérianité et artinianité. Signalons tout de suite qu'il est facile de montrer qu'une algèbre topologique vérifie $\left(\mathrm{F}_{\mathrm{g}}\right)$ si, et seulement si, son unitisée la vérifie. Ceci permettra toujours de se placer dans le cas unitaire.

Nous commençons par le résultat suivant qui caractérise la $Q$-propriété pour une classe de $F$-algèbres.

Proposition 1. Soit $A$ une F-algèbre dont la famille des idéaux à gauche principaux satisfait la condition de la chaine ascendante. Alors les assertions suivantes sont équivalentes :

(1) A est une $Q$-algèbre;

(2) $(\forall x \in A, \overline{A x}=A) \Leftrightarrow x \in G(A)$.

Preuve. $(1) \Rightarrow(2)$. Soit $x \in A$ tel que $\overline{A x}=A$. Par l'assertion (1), on doit avoir $A x=A$. Il en résulte que $x$ est inversible à gauche dans $A$. D'après $[6$, Théorème 1, p. 353], $x$ est inversible dans $A$.

$(2) \Rightarrow(1)$. Supposons que $A$ n'est pas une $Q$-algèbre. Il existe alors une suite $\left(x_{n}\right)$ d'éléments non inversibles de $A$ tel que $x_{n} \rightarrow e$ quand $n \rightarrow \infty$. En raisonnant comme dans [5], on construit une suite $\left(e_{n}\right)$ d'éléments de $A$ vérifiant : (i) $e_{n} \rightarrow e$ quand $n \rightarrow \infty$; (ii) $\forall n, e_{n}=x_{n} e_{n+1}$. (L'hypothèse de commutativité et de $m$-convexité supposée dans [5] n'intervient pas dans la construction de la suite $\left(e_{n}\right)$.) L'hypothèse faite sur $A$ entraîne l'existence d'un entier $n_{0}$ tel que $A e_{n}=A e_{n_{0}}$ pour tout $n \geq n_{0}$. Il en résulte que $\overline{A e_{n_{0}}}=A$. Par l'assertion (2), $e_{n_{0}}$ est inversible à gauche dans $A$ et donc 
inversible via $\left[6\right.$, théorème 1, p. 353]. Ainsi, l'élément $x_{n_{0}}$ est inversible à droite dans $A$ et donc inversible par [6, théorème 1, p. 353], ce qui n'est pas le cas.

Voici maintenant une caractérisation de la propriété $\left(\mathrm{F}_{\mathrm{g}}\right)$ dans la classe des $F$-algèbres.

ThÉorème 2. Soit A une F-algèbre. Les assertions suivantes sont équivalentes :

(1) A vérifie $\left(\mathrm{F}_{\mathrm{g}}\right)$;

(2) A satisfait les deux assertions suivantes:

(a) A est noethérienne à gauche,

(b) A est une $Q$-algèbre.

Preuve. $(1) \Rightarrow(2)$. Soit $\left(I_{n}\right)$ une suite croissante d'idéaux à gauche de $A$. Posons $I=\bigcup_{n} I_{n}$. C'est un idéal à gauche fermé de $A$. Par le théorème de Baire, il existe $n_{0}$ tel que $I_{n_{0}}$ est d'intérieur non vide dans $I$. Il en résulte que $I=I_{n_{0}}$ et par suite $I_{n}=I_{n_{0}}$ pour tout $n \geq n_{0}$. La deuxième assertion découle de la proposition précédente.

$(2) \Rightarrow(1)$. Soit $\mathcal{F}$ la famille des idéaux à gauche non fermés de $A$. Supposons qu'elle est non vide et considérons un élément maximal $I$ dans $\mathcal{F}$. Soit $x \in \bar{I}$ avec $x \notin I$. On a $\bar{I}=I+A x$. Considérons $x_{1}, \ldots, x_{r} \in I$ tel que $I=A x_{1}+\cdots+A x_{r}$, et $J=\{a \in A: a x \in I\}$ qui est un idéal à gauche de $A$. Soit $\varphi: A^{r+1} \rightarrow \bar{I}$ avec

$$
\varphi\left(a_{1}, \ldots, a_{r+1}\right)=\sum_{1 \leq i \leq r} a_{i} x_{i}+a_{r+1} x .
$$

L'application $\varphi$ est linéaire continue et surjective. Elle est alors ouverte. Si $O$ est un ouvert non vide de $A^{r+1}$, il existe $a_{1}, \ldots, a_{r}, a_{r+1} \in O$ tel que $\sum_{1 \leq i \leq r} a_{i} x_{i}+a_{r+1} x \in I$. Donc $a_{r+1} x \in I$. Il en résulte que $a_{r+1} \in J$ et par suite que $J$ est dense dans $A$. Comme $A$ est une $Q$-algèbre, on a $J=A$. Donc $x \in I$, ce qui n'est pas le cas.

REMARQUES. (1) La métrisabilité est nécessaire pour la validité du théorème précédent. En effet, voici un exemple d'une $Q$-a.l.m.c. complète commutative dont tout idéal est fermé et qui n'est pas noethérienne. Pour tout entier naturel $k \geq 1$, considérons $A_{k}=\left\{\left(x_{n}\right) \in S_{0}^{1}: \forall n \geq k, x_{n}=x_{k}\right\}$. Les $A_{k}$ forment une suite croissante de sous-algèbres de dimension finie de $S_{0}^{1}$. De plus $S_{0}^{1}=\bigcup_{k \geq 1} A_{k}$. Munie de la topologie localement convexe limite inductive, l'algèbre $S_{0}^{1}$ est une a.l.m.c [2, proposition 11, p. 349]. Elle est complète et, de plus, elle est tonnelée puisque les $A_{k}$ le sont. D'autre part, le spectre de tout élément de $S_{0}^{1}$ est borné. Donc, d'après [9, corollaire 3, p. 296], $S_{0}^{1}$ est une $Q$-algèbre. Cependant, $S_{0}^{1}$ n'est pas noethérienne. 
Par ailleurs, il est facile de voir que tout idéal propre de $S_{0}^{1}$ est égal à l'intersection des idéaux maximaux le contenant. Il en résulte que l'algèbre $S_{0}^{1}$ vérifie $\left(\mathrm{F}_{\mathrm{g}}\right)$.

(2) Dans le cas commutatif, une $F$-algèbre commutative noethérienne est une $Q$-algèbre. Voir [4] ou [8].

Il découle d'un résultat d'Arens que, dans une a.l.m.c. de Fréchet, l'adhérence d'un idéal à gauche, de type fini, propre est également propre. D'où, dans le cas Fréchet, l'équivalence suivante qui est une extension au cas non commutatif du théorème 4 de [8, p. 298].

ThÉonème 3. Pour qu'une a.l.m.c. de Fréchet vérifie $\left(\mathrm{F}_{\mathrm{g}}\right)$, il faut, et il suffit, qu'elle soit noethérienne à gauche.

Preuve. Par le théorème 2, on sait que la condition $\left(\mathrm{F}_{\mathrm{g}}\right)$ implique la noethérianité. Pour la réciproque, il suffit, par le même théorème, de montrer que $A$ est une $Q$-algèbre. Pour cela, on utilise la deuxième assertion de la proposition 1. En effet, si $\overline{A x}=A$ pour $x \in A$, alors par un résultat d'Arens ([1, Theorem 3.2, p. 173]), on a aussi $A x=A$, i.e., $x$ est inversible à gauche. Donc $x$ est inversible, puisqu'on est dans le cas noethérien ([6, théorème 1 , p. 353]).

L'algèbre $S_{0}^{1}$ possède la propriété suivante: Pour tout idéal $I$ de $S_{0}^{1}$, il existe une partie $J$ de $S_{0}^{1}$ et $x \in I$ tel que $I=\operatorname{Ann}(J)+\mathbb{C} x$, où $\operatorname{Ann}(J)=$ $\left\{x \in S_{0}: x y=0, \forall y \in J\right\}$. Il en résulte que $S_{0}^{1}$ vérifie $\left(\mathrm{F}_{\mathrm{g}}\right)$ pour toute topologie d'algèbre. Nous allons montrer que c'est également le cas pour les algèbres commutatives artiniennes, en particulier, les algèbres qui sont des produits finis d'algèbres qui sont des corps. Dans le cas commutatif, nous caractérisons ces dernières dans la classe des algèbres noethériennes semi-simples.

ThÉORÈme 4. Soit A une algèbre commutative artinienne. Alors A vérifie $\left(\mathrm{F}_{\mathrm{g}}\right)$ pour toute topologie d'algèbre.

Preuve. Soit $\tau$ une topologie d'algèbre sur $A$ et $\mathcal{F}$ la famille des idéaux principaux de $A$ qui ne sont pas fermés. Supposons que $\mathcal{F}$ n'est pas vide et considérons un élément $A x_{0}$ minimal de $\mathcal{F}$. Comme $A x_{0}$ n'est pas fermé, on a $A x_{0}^{2} \neq A x_{0}$. Vu le caractère minimal de $A x_{0}$, l'idéal $A x_{0}^{2}$ est fermé. Par ailleurs, l'algèbre $A x_{0} / A x_{0}^{2}$, dont le produit est trivial, est artinienne. Elle est alors de dimension finie. L'idéal $A x_{0} / A x_{0}^{2}$ est donc fermé dans l'algèbre topologique quotient $A / A x_{0}^{2}$. Il en résulte que l'idéal $A x_{0}$ est fermé dans $A$, ce qui n'est pas le cas. Ainsi $\mathcal{F}$ est vide. Donc tout idéal principal de $A$ est fermé. Comme l'artinianité et la propriété $\left(\mathrm{F}_{\mathrm{g}}\right)$ sont preservées par passage au quotient, tout idéal de type fini de $A$ est fermé. Donc $A$ vérifie $\left(\mathrm{F}_{\mathrm{g}}\right)$ vu qu'elle est noethérienne [7, théorème IV.4, p. 68]. 
La réciproque du théorème précédent est fausse. Cependant, le résultat suivant en fournit une réciproque. Rappelons d'abord qu'un idéal premier d'une algèbre commutative $A$ est dit minimal s'il ne contient aucun idéal premier de $A$ autre que lui-même.

THÉORÈme 5. Soit $A$ une algèbre commutative semi-simple noethérienne $A$. Les conditions suivantes sont équivalentes:

(1) A vérifie $\left(\mathrm{F}_{\mathrm{g}}\right)$ pour toute topologie d'algèbre;

(2) A est isomorphe à un produit fini d'algèbres qui sont des corps;

(3) A est artinienne.

Preuve. Les implications $(2) \Rightarrow(1)$ et $(2) \Rightarrow(3)$ sont faciles. L'implication $(3) \Rightarrow(2)$ découle de $[7$, corollaire, p. 67] et de la semi-simplicité de $A$. Montrons que $(1) \Rightarrow(2)$. Soit $\left(M_{i}\right)_{i \in I}$ la famille des idéaux maximaux de $A$. Si $I$ est réduit à un seul point alors l'algèbre $A$ est un corps vu qu'elle est semi-simple. Supposons maintenant que $I$ n'est pas un singleton. Soit $i_{0} \in I$ et posons $L=\bigcap_{i \neq i_{0}} M_{i}$. Supposons que $L$ est nul. On munit $A$ de la topologie définie par la famille de semi-normes $\left(p_{i, f}\right)_{i \in I \backslash\left\{i_{0}\right\}, f \in\left(A / M_{i}\right)^{*} \text {, avec }}$ $p_{i, f}(x)=\left|f\left(s_{i}(x)\right)\right|$ pour tout $x \in A$, où $s_{i}$ est la surjection canonique de $A$ sur $A / M_{i}$, et $\left(A / M_{i}\right)^{*}$ est le dual algébrique de $A / M_{i}$.

Remarquons que, pour tous $i \in I$ et $f \in\left(A / M_{i}\right)^{*}$, le noyau de $p_{i, f}$ contient l'idéal $M_{i}$. Montrons qu'il existe $J$ fini contenu dans $I \backslash\left\{i_{0}\right\}$ tel que $M_{i_{0}}$ contient $\bigcap_{j \in J} M_{j}$. Dans le cas contraire, pour toute partie finie $J$ de $I \backslash\left\{i_{0}\right\}$, il existe $x_{J} \in \bigcap_{j \in J} M_{j}$ tel que $x_{J} \notin M_{i_{0}}$. On a alors $M_{i_{0}}+A x_{J}=A$. Donc, il existe $a_{J} \in A$ et $m_{J} \in M_{i_{0}}$ tel que $e=m_{J}+a_{J} x_{J}$. Or la suite généralisée $\left(a_{J} x_{J}\right)_{J}$ converge vers zéro, et donc la suite $\left(m_{J}\right)_{J}$ converge vers $e$, ce qui est impossible vu que $M_{i_{0}}$ est fermé.

Ainsi, il existe $J$ fini contenu dans $I \backslash\left\{i_{0}\right\}$ tel que $M_{i_{0}}$ contient $\bigcap_{j \in J} M_{j}$. Par suite, il existe $j \in J$ tel que $M_{i_{0}}=M_{j}$, ce qui ne peut être le cas. Donc nécessairement $L$ est non nul. De plus, on a $M_{i_{0}} \cap L=\{0\}$. Comme $A$ est semi-simple, il existe un idéal premier minimal $P$ de $A$ ne contenant pas $L$; l'idéal $P$ contient alors $M_{i_{0}}$ et par suite $P=M_{i_{0}}$. On conclut par [7, corollaire, p. 106]. Finalement, $I$ est fini et $A$ est isomorphe à l'algèbre produit $\prod_{i \in I} A / M_{i}$.

\section{Références}

[1] R. Arens, Dense inverse limit rings, Michigan Math. J. 5 (1958), 169-182.

[2] A. Arosio, Locally convex inductive limits of normed algebras, Rend. Sem. Mat. Univ. Padova 51 (1974), 333-359.

[3] G. Carboni and A. Larotonda, An example of a Fréchet algebra which is a principal ideal domain, Studia Math. 138 (2000), 265-275.

[4] R. Choukri and A. El Kinani, Topological algebras with ascending or descending chain condition, Arch. Math. (Basel) 72 (1999), 438-443. 
[5] J. Esterle, Idéaux maximaux denses dans les algèbres de Fréchet, Bull. Sci. Math. 119 (1995), 187-194.

[6] N. Jacobson, Some remarks on one-sided inverses, Proc. Amer. Math. Soc. 1 (1950), $352-355$.

[7] J. P. Lafon, Algèbre commutative. Langages géométrique et algébrique, Hermann. Paris, 1977.

[8] W. Żelazko, A characterization of commutative Fréchet algebras with all ideals closed, Studia Math. 138 (2000), 293-300.

[9] -, On maximal ideals in commutative m-convex algebras, ibid. 58 (1976), 290-298.

Département de Mathématiques

École Normale Supérieure

B.P. 5118

Rabat, 10105, Maroc

E-mail: rchoukri@hotmail.com

abdellah_elkinani@hotmail.com

moudadess@hotmail.com

Received June 14, 2004

Final version December 14, 2004 